\title{
DYNAMIC PROPERTIES OF AQUEOUS FOAMS
}

\section{Von Hippel Award Address}

The following is the talk presented Nov. 3 in Boston by Prof. Clarence C. Zener, winner of the Society's Von Hippel Award for outstanding achievement in materials science.

I am particularly honored to receive the Von Hippel Award from the Materials Research Society. Von Hippel's and my path crossed 49 years ago. We both published papers on the same topic, the electrical breakdown of crystals. Some 18 years later the Bell Laboratories invented the semiconductor diode, and named it after me, attributing its breakdown to the mechanism I had described. Later they found that under many conditions the diode breakdown is due to the mechanism described by von Hippel. Ever since then I have admired the insight which von Hippel applied to engineering materials. I wish he could be here tonight to hear how, with the proper understanding, one can persuade the most common material of all, namely water, to convert automatically its thermally stored enthalpy into useful work without the usual mechanical accessories of heat exchangers and gas turbines.

My wife was horrified when she saw the title to this talk. "You should call it, 'Bubbles, Bubbles, Toil and Trouble," she said. For some years all she heard me talk about were bubbles. In our lab a four-inch diameter column extended upwards three floors high. It contained 1,100 capillary tubes. Each tube emitted 20 bubbles per second. As each bubble rose, it expanded and transformed from a spherical bubble into a polyhedral cell of a foam. As the foam continued to rise, the individual cells became larger and larger, and rose at ever increasing speeds. I shall describe now the concepts which led to the construction of this column, and which led us to believe that a larger diameter tube several hundred feet high would lead us to an economic way of converting

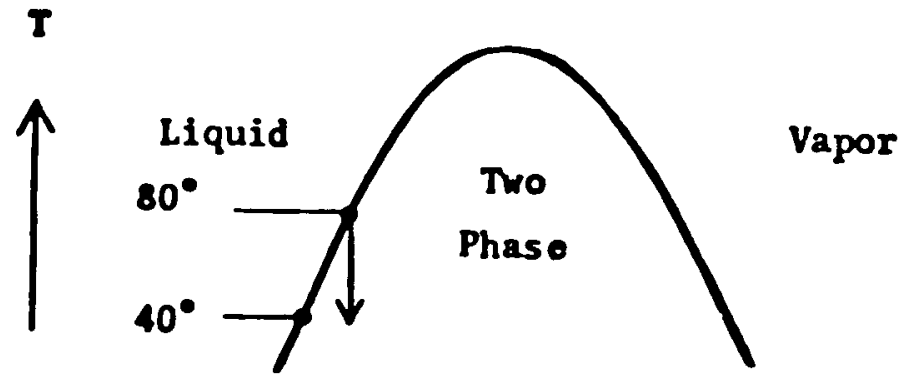

Fig. 1 Temperature-enthalpy diagram of water.

thermally stored enthalpy into useful work.

I can vividly present these concepts in terms of the T-S diagram for water, Fig. 1. To the left of the T-S curve water is a liquid, to the right it is a vapor. Below the T-S curve the water is a mixture of liquid and vapor. I now address the following question. Suppose we have a large heat reservoir at a subambient temperature, say $40^{\circ} \mathrm{F}$. Near this reservoir we have a supply of room temperature water, namely $80^{\circ} \mathrm{F}$. How can we take advantage of the higher enthalpy of this $80^{\circ} \mathrm{F}$ water to obtain power without having to employ expensive heat exchangers? Our answer to this question was to establish a rising foam column. This we would do by feeding into the column our $80^{\circ} \mathrm{F}$ water in the form of a close packed array of bubbles at the saturation vapor pressure of $80^{\circ} \mathrm{F}$ water. As an element of this close packed array rises, several changes take place simultaneously: the pressure drops, the temperature also drops, evaporation takes place, the foam cells expand. In the absence of losses, the foam will rise 960 feet before the pressure drops to the saturation pressure of the $40^{\circ} \mathrm{F}$ water.
If we break the foam at this height, the freed vapor can be conducted back to the ground level, there to be condensed by a spray of $40^{\circ} \mathrm{F}$ water. The water from the broken foam is collected into a pipe, which in turn feeds it into a hydraulic turbine at ground level, thereby providing a 960 foot head. The practical application we have in mind is for the Gulf of Mexico or other tropical ocean water. Here the top mixed layer of 200 feet depth is our pool of $80^{\circ} \mathrm{F}$ water. The cold deep water provides the $40^{\circ} \mathrm{F}$ reservoir.

A remarkable feature of foam is its mechanical temporary stability. This stability requires, however, a small quantity of surfactant. In order to understand the role played by surfactants, consider one foam cell, Fig. 2. Each of the six corners is bounded by convex boundaries. Because of their surface tension, convex boundaries produce a lower pressure inside the corners than in the vapor. However, the pressure between the flat walls is identical to the pressure in the surrounding vapor. The pressure within the corners is therefore less thar the pressure within the walls. In the absence of intervening forces, the corners would therefore spontaneously 


\section{AQUEOUS FOAMS}

pull the walls apart.

Willard Gibbs pointed out in the 1870 s that an intervening force is provided by molecules that are repelled from the interior of the water onto the surface. These molecules are now called surfactants. Solute molecules in the interior of water exert a pressure, called osmotic pressure, which is numerically equal to the pressure they would exert on the walls of a container if they were a gas. Likewise these surfactant molecules exert a twodimensional spreading pressure of the same magnitude as if they were gas molecules confined to a surface. As the walls first start being sucked into the corners, an excess of surfactant molecules builds up on the corner surfaces. It is the excess spreading pressure of these surfactant molecules that obstruct further drag of the wall surfaces towards the corner. The walls therefore behave as if their surfaces were incompressible. Because they themselves remain stationary, they retard the flux of the interior water into the corners. This flux of the interior water into the corners results in a gradual thinning of the walls. Because of the limited time of several seconds that a given foam cell remains in our 30 foot column, the cells walls never get thinner than 1 micron. They are one to two orders of magnitude thicker than the walls of foam commonly investigated in the laboratory. We thus speak of our foam as wet foam.

Contrary to the diversity that prior investigators had found with mature "dry" foams, we found our wet foams obey a very simple flow law. This is the Navier-Stokes flow law for a homogeneous fluid, combined with the equation of state of an isentropic liquid-vapor mixture. For example, the drop in temperature with height agrees precisely with the calculated value. Completely unanticipated, however, was the periodic collapse of the foam column when we reduced the surfactant concentration below $100 \mathrm{ppm}$. This concentration was more than an order of magnitude higher than our calculated value for the maintenance of foam stability. This foam collapse followed a regular pattern. Seconds before the collapse the pressure at the bottom of the column would rise, and [Continued on Page 10]

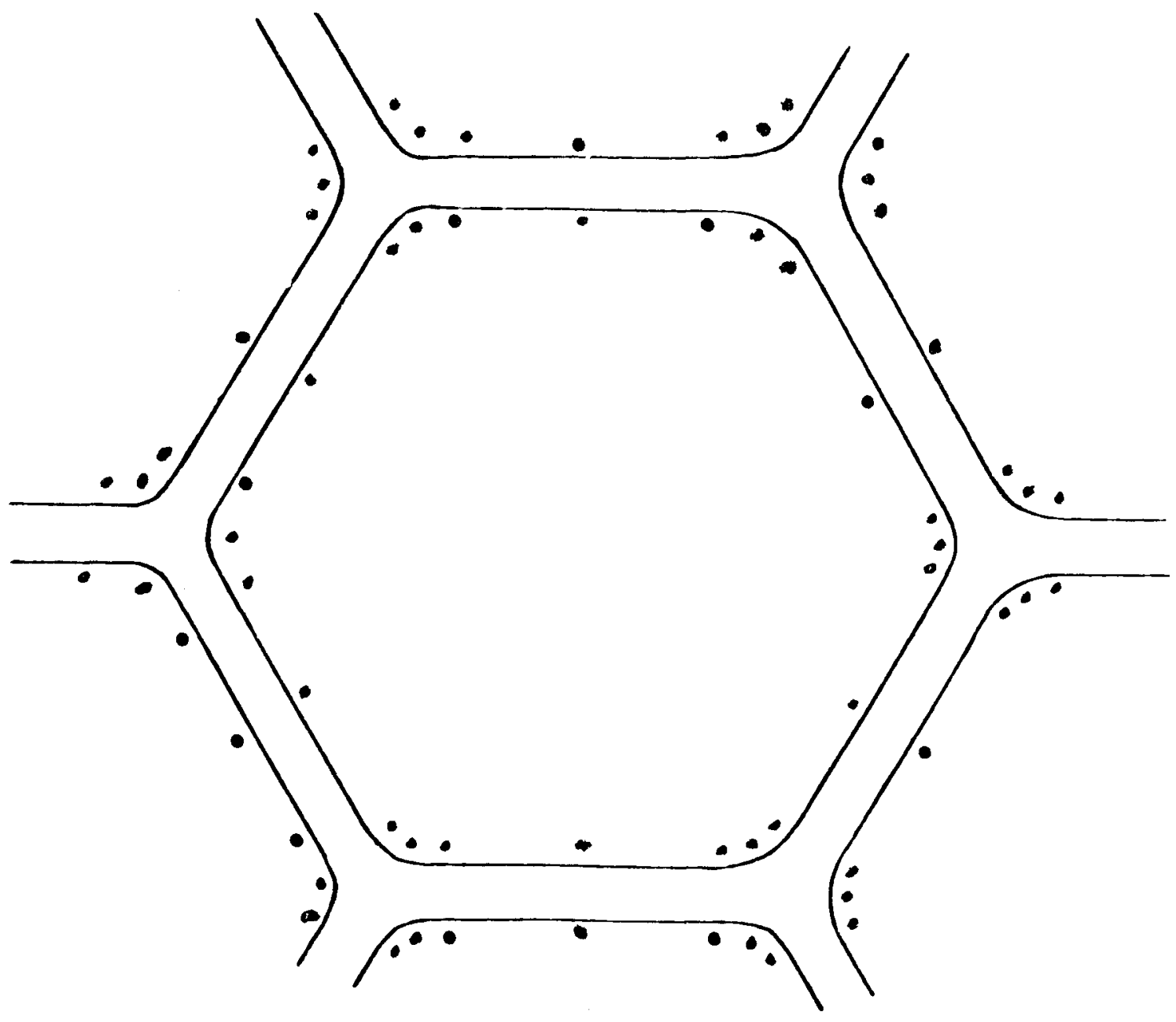

Fig. 2 Distribution of surfactant molecules on a foam surface. 


\section{AQUEOUS FOAMS}

\section{[Continued from Page 9]}

the exit velocity at the top of the column would rise to perhaps twice its normal value, from 10 to 20 feet per second.

A clue as to what was happening was found in Lord Rayleigh's book, The Theory of Sound, written in the last century. Being an old fashioned scientist, Lord Rayleigh blew his own glassware. He observed that when he closed the end of a tube with a blow torch, he heard a loud note whose quarter wave length equaled the length of the tube. This sound lasted as long as the closed end remained hot. Being no mere technician, Rayleigh gave a lucid interpretation:

Consider a vibrating element of volume [he wrote]. As it vibrates up and down, it adiabatically expands and compresses. It therefore suffers adiabatic cooling and heating. When it is compressed, and hence warmer than normal, it is close to the closed hot end. Heat hence flows from the hot tube to the not-so-hot air. When the gas expands, and is hence cooler than normal, it is close to the open cold end, and hence finds itself surrounded by a still cooler tube. Our element of air now loses the heat it had acquired at the hot end. Our air element thus acquires heat at a high temperature, discards this heat at a low temperature, and therefore acts as a heat engine. The work which it thereby acquires goes into increasing its amplitude of vibration.

The basic lesson that we learn from Lord Rayleigh is that a tube of vapor which has a hot closed end and a cold open end is not necessarily in a stable condition. What intrigued us was that our rising foam column has precisely these conditions. The bottom is filled with water, and is therefore a close end from the viewpoint of the foam. It is at room temperature. The upper end is open to the condenser, and is below room temperature. This foam-filled tube thus satisfies the Rayleigh conditions. The outstanding question remains: Why is our foam column unstable only at low surfactant concentrations?

In order to answer this question we must obtain a quantitative measure of the degree of instability. If the air within the Rayleigh tube were such a poor heat conductor that no appreciable amount of heat could flow between the air and the tube walls, the air vibrations would be adiabatic, and no amplification could take place. On the other hand, if the thermal conductivity were so high that the air were always at the same temperature as the adjacent tube walls, the air would be isothermal with respect to the walls. Again no amplification would take place. Only when we are in the intermediate range, neither adiabatic nor isothermal, can we expect appreciable damping. The appropriate ratio that tells us the susceptibility to amplification is the adiabatic ratio $f d^{2} / D$

where $f$ is the frequency of vibration, $d$ the tube diameter and $D$ the thermal diffusivity,

$D=k / \rho c$

where $\mathrm{k}$ is thermal conductivity, $\rho$ the density and $c$ the specific heat. Very low values of this ratio gives isothermal vibration with no amplification; very high values give adiabatic vibrations with no amplification. Only intermediate values in the vicinity of unity give appreciable amplification.

Our first estimate of the adiabatic ratio gave a very high value, in the range $1,000-10,000$, way in the extreme adiabatic range. This comes from the very low thermal diffusivity of the vapor within the foam cells. However, thermal conduction within a foam cell may be by the heat pipe effect. Here we have evaporation on the cell wall which is at the higher temperature, condensation on the opposite cell wall at a lower temperature. Heat flows by convection of the vapor from the warm evaporating surface to the cool condensing surface. The thermal conductivity by this heat pipe effect is 10,000 greater than by thermal diffusivity. We are now in the amplifying range.

At high surfactant concentrations the cell walls within the foam are completely covered by surfactant molecules. The heads of these molecules are submerged in the water, their long hydrocarbon tails are sticking out of the surface, providing a thick mat which greatly retards both evaporation and condensation. At $>100 \mathrm{ppm}$ the solution has enough surfactant molecules to keep the foam surfaces completely covered. Evaporation from the surface is now greatly retarded. Vibrations cannot be amplified. at $<100 \mathrm{ppm}$, the expanding foam surface thins the covering surfactant mat, rendering it now permeable to water vapor. The resulting 10,000-time increase in the thermal conductivity throws the adiabatic ratio into the region of high amplification. Just as over damping gives rise to an exponential decay in velocity, so the over amplification gives an exponential increase in velocity. The foam literally explodes. Now that we understand the origin of this explosion, we know how to operate such a foam column free of explosions, even at low surfactant concentrations. We have merely to maintain the walls of the column at a slightly lower temperature than the foam itself.

\section{References}

Clarence Zener and John Fetkovich, Science 189, 294 (1975).

Clarence Zener and Martin Greenstein, Energy 5, 503 (1980).

Clarence Zener and Heime Noriega, Proc. Natl. Acad. Sci. 79, 3384 (1982).

\section{Materials Scientist}

Company in Southern California seeks Material Scientist with experience in laser processing of silicon to develop applications in semiconductor and photovoltaic manufacturing. Please call (714) 675-5270 or write:

Phillip D'Aigle

Alan Glenn, Inc.

2711 E. Coast Hwy., Suite 203

Corona Del Mar, CA 92625 